\title{
Performance of multi-aperture grid extraction systems for an ITER-relevant RF-driven negative hydrogen ion source
}

\author{
P. Franzen, R. Gutser, U. Fantz, W. Kraus, H. Falter, M. Fröschle, \\ B. Heinemann, P. McNeely, R. Nocentini, R. Riedl, A. Stäbler and \\ D. Wünderlich
}

Max-Planck-Institut für Plasmaphysik (IPP), EURATOM Association, D-85748 Garching, Germany

E-mail: peter.franzen@ipp.mpg.de

Published in Nucl. Fusion 51 (2011) 073035 (13pp) doi:10.1088/0029-5515/51/7/073035

\begin{abstract}
The ITER neutral beam system requires a negative hydrogen ion beam of $48 \mathrm{~A}$ with an energy of $0.87 \mathrm{MeV}$, and a negative deuterium beam of $40 \mathrm{~A}$ with an energy of $1 \mathrm{MeV}$. The beam is extracted from a large ion source of dimension $1.9 \times 0.9 \mathrm{~m}^{2}$ by an acceleration system consisting of seven grids with 1280 apertures each. Currently, apertures with a diameter of $14 \mathrm{~mm}$ in the first grid are foreseen.

In 2007, the IPP RF source was chosen as the ITER reference source due to its reduced maintenance compared with arc-driven sources and the successful development at the BATMAN test facility of being equipped with the small IPP prototype RF source ( $\sim \frac{1}{8}$ of the area of the ITER NBI source). These results, however, were obtained with an extraction system with $8 \mathrm{~mm}$ diameter apertures.

This paper reports on the comparison of the source performance at BATMAN of an ITER-relevant extraction system equipped with chamfered apertures with a $14 \mathrm{~mm}$ diameter and $8 \mathrm{~mm}$ diameter aperture extraction system. The most important result is that there is almost no difference in the achieved current density-being consistent with ion trajectory calculations - and the amount of co-extracted electrons. Furthermore, some aspects of the beam optics of both extraction systems are discussed.
\end{abstract}

(Some figures in this article are in colour only in the electronic version)

\section{Introduction}

The IPP RF-driven caesiated negative hydrogen ion source is now the reference source for the ITER neutral beam system [1-3] due to the reduced maintenance compared with arc-driven sources-only the Cs supply has to be changed periodically — and due to the successful development over the last decade [4-8]. Accelerated current densities of $330 \mathrm{~A} \mathrm{~m}^{-2}$ with $\mathrm{H}^{-}$and $230 \mathrm{~A} \mathrm{~m}^{-2}$ with $\mathrm{D}^{-}$have been achieved with the small IPP RF prototype source $\left(\sim \frac{1}{8}\right.$ of the area of the ITER NBI source) on the test facility BATMAN (Bavarian Test Machine for Negative Ions) at the required source pressure of $0.3 \mathrm{~Pa}$. Also demonstrated was that the current of co-extracted electrons was less than the extracted ion current, as required by ITER. The extraction area was small $\left(0.007 \mathrm{~m}^{2}\right)$ and the pulse length limited to $4 \mathrm{~s}$.

The record values at BATMAN were obtained with an extraction system with optimized aperture geometry, i.e. using chamfered apertures; the aperture diameter $(8 \mathrm{~mm})$ in the first grid was smaller than that foreseen currently for the ITER extraction system (14 mm diameter). This difference was not regarded as a major concern until now: preliminary results at BATMAN with the two different multi-aperture extraction systems available at IPP showed no major dependence of the extracted current density on the aperture diameter [4]. The experiments with the $14 \mathrm{~mm}$ aperture extraction system, however, were hampered by technical problems-weak power load handling capability leading to several water leaks - and by a non-optimized aperture geometry, i.e. flat apertures, so that a clear conclusion could not be drawn.

Meanwhile, experiments at the SINGAP test facility (CEA Cadarache) with a filamented source showed that for single aperture extraction, the achievable current density does depend on the aperture diameter [9] for caesiated conditions. For the $8 \mathrm{~mm}$ diameter aperture compared with the $14 \mathrm{~mm}$ diameter aperture, the accelerated current density increased by a factor of almost two for the same source conditions. As these results would have consequences for the design of the ITER extraction 


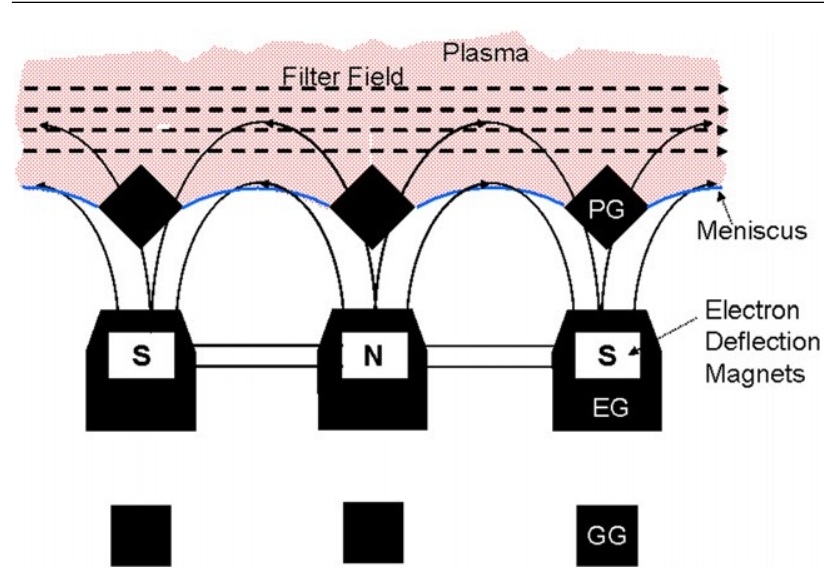

Figure 1. Sketch of the extraction region and the extraction system of a negative hydrogen ion source. The PG is at a high negative potential, the extraction voltage, i.e. the voltage between the plasma and EG, is $5-10 \mathrm{kV}$. At the IPP test facilities, the filter field is perpendicular to the field of the electron deflection magnets.

system, new experiments have been performed at BATMAN with an upgraded $14 \mathrm{~mm}$ aperture extraction system, i.e. with improved power load handling capabilities and also equipped with chamfered apertures. As the detailed grid geometry of the ITER system is still under some discussion, experiments with two different extraction gaps ( 3 and $6 \mathrm{~mm}$ ) have been performed. The $6 \mathrm{~mm}$ gap is currently the ITER NBI design value.

This paper concentrates mainly on the comparison of the achieved current densities and the amount of coextracted electrons of the $14 \mathrm{~mm}$ aperture extraction system with the $8 \mathrm{~mm}$ aperture system. Finally, the beam optics properties of the different extraction systems are also discussed.

\section{Experimental background}

\subsection{Extraction region in caesiated negative hydrogen ion sources}

Figure 1 shows a sketch of the geometry of the extraction region of a negative hydrogen ion source. In the case of the IPP test facilities with moderate total voltages of up to a few $10 \mathrm{kV}$, the extraction system consists of three grids: the plasma grid (PG) facing the plasma, the extraction grid (EG) and the grounded grid (GG)

A magnetic field in front of the PG is necessary to minimize the amount of co-extracted electrons-the coextracted electrons are deflected out of the beam onto the EG by magnets embedded in the EG-and to enhance the extraction probability of the surface produced negative ions in caesiated negative hydrogen ion sources. One main mechanism for the enhancement of the extraction probability by the filter field is the reduction of the electron temperature to below $2 \mathrm{eV}$, which is necessary for minimizing the destruction rate of the negative hydrogen ions by electron collisions; then mutual neutralization with positive ions takes over as the dominant destruction process.

The generation of sufficient negative hydrogen ions in ITER-relevant ion sources faces different challenges that have to be fulfilled simultaneously.

- In order to achieve the required accelerated ion currents (48 $\mathrm{A}$ at $870 \mathrm{keV}$ in hydrogen, $40 \mathrm{~A}$ at $1 \mathrm{MeV}$ in deuterium), the source has to deliver negative hydrogen currents of up to $70 \mathrm{~A}$ due to the stripping losses in the accelerator. (The required extracted current ratings are $70 \mathrm{~A} \mathrm{H}^{-}$for the diagnostic neutral beam injector, $68 \mathrm{~A} \mathrm{H}^{-}$ and $59 \mathrm{~A} \mathrm{D}^{-}$for the heating neutral beam injectors $[2,3]$.) In order to minimize the stripping losses, i.e. losses of negative hydrogen ions due to collisions with the residual gas in the extraction system, the source must be operated at a low source pressure of $0.3 \mathrm{~Pa}$; at this pressure the stripping losses are calculated to be still $25-30 \%$ in the ITER NBI system [10].

- The unavoidable co-extracted electrons must be deflected out of the beam at moderate extraction voltages. The power handling of the EG is the main technical limitation of negative hydrogen sources and requires the amount of co-extracted electrons to be less than the extracted ion current and the extraction voltage to be $10-12 \mathrm{kV}$ at maximum - this is about one order of magnitude lower than the extraction voltage in positive ion based neutral beam systems. Even when these conditions are fulfilled, a power density of up to $30 \mathrm{MW} \mathrm{m}^{-2}$ can be achieved on small spots on the EG [11].

From the experiences obtained in the last three decades in different experiments worldwide [4-6, 12-16], the maximum achievable current density under these extreme conditionslow pressure, low extraction voltage, low amount of co-extracted electrons - is in the range of 200-300 $\mathrm{A} \mathrm{m}^{-2}$ for deuterium and 300-400 $\mathrm{A} \mathrm{m}^{-2}$ for hydrogen (as the amount of co-extracted electrons is much lower in hydrogen, a larger ion current density can be achieved by reducing the filter field strength). These high negative ion current densities could only be achieved until now with caesium seeding of the source. Caesium forms a layer with a low work function at the source surfaces on which impinging hydrogen neutrals and ions are converted to negative ions; these are accelerated back into the plasma by the sheath potential. As the binding energy of the electron is rather low $(0.75 \mathrm{eV})$, the mean free path of a negative hydrogen ion in the main source plasma with an electron temperature of $5-10 \mathrm{eV}$ [6] is only in the range of a few centimetres [17]. Hence, only negative ions created near the PG apertures can be extracted, but they have to be bent back to the apertures by magnetic fields and charge exchange collisions with hydrogen neutrals [18]. The typical negative ion density in fusion-relevant ion sources near the meniscus is several $10^{17} \mathrm{~m}^{-3}$ [19].

As a consequence of the achieved negative ion current densities, the source for the ITER NBI system must be quite large in order to deliver the required ion current; currently foreseen is a source of size $1.9 \times 0.9 \mathrm{~m}^{2}$ with 1280 apertures of $14 \mathrm{~mm}$ diameter corresponding to an extraction area of $0.2 \mathrm{~m}^{2}$. Together with the necessary filter field and caesium seeding of the source, this large source dimension is still a challenge for homogeneous production of negative ions across the PG, which is necessary for good beamlet transmission through the apertures of the extraction and acceleration grids. The homogeneity of such large RF sources is not proven up 
to now and is part of the NBI R\&D programme in Europe for the next few years [20], consisting of the planned ELISE test facility at IPP for large-scale extraction from a half-size ITER source [21, 22] and the PRIMA test facilities currently designed by Consorzio RFX in Padua, Italy. ELISE is an important intermediate step with large experimental flexibility between the small prototype source extraction experiments at IPP Garching and the full size ITER source [23].

The amount of co-extracted electrons is reduced by the aforementioned magnetic filter field across the ion source, together with biasing the PG positively against the source itself. In the RF source, the optimum bias voltage is near the floating potential of the grid $(\sim 10 \mathrm{~V})$ so that only a small bias current can flow $[4,5,24]$. Additionally, the effect of the PG bias must be supported by a so-called bias plate near the PG for an effective suppression of the amount of co-extracted electrons, especially for deuterium operation [25].

The boundary between the field-free plasma and the region where charged particles feel the extraction voltage is called the 'meniscus' as it forms a curved surface across the PG aperture. Hence, the extraction potential is zero at the meniscus. Apart from the grid geometry, the geometry of the meniscus is determined by the space charge of the plasma particles, i.e. by the density and the extraction potential. The meniscus forms a convex lens and so determines the initial beamlet quality. For positive ion extraction, where the space charge of the positive ions can be easily compensated by the fast electrons, the dependence of the meniscus shape on the plasma parameters and the extraction potential is well understood; for negative ion extraction, however, the situation is more complex as the space charge of the negative ions must be compensated by slow positive ions $\left(\mathrm{H}^{+}, \mathrm{H}_{2}^{+}, \mathrm{H}_{3}^{+}\right.$, but also $\mathrm{Cs}^{+}$in the case of caesiated sources). The electrons do not contribute to the space charge at the meniscus, as here the electron density must be much smaller than the negative ion density-a factor of 42 if the ion and electron temperatures are the same-in order to have, as required for an ITER-relevant ion source, the coextracted electron current less than the extracted negative ion current.

Due to the embedded magnets, the EG has to be rather thick ( $\sim 10 \mathrm{~mm})$. Together with the low extraction voltagecompared with positive ion extraction-this is a drawback for the ion optics as due to the relative low ion velocity, the beamlets are expanded within the potential free EG by the space charge.

For caesium seeded negative hydrogen ion sources, the increase in the ion yield during the build-up of the caesium layer at the PG is accompanied by a decrease in the amount of co-extracted electrons. This is commonly explained by a reduction in the electron density by the negative charge produced by the ions near the PG in order to maintain the quasineutrality of the plasma. Finally, a so-called boundary layer is formed near the PG, extending a few centimetres into the source where the negative hydrogen ions are the dominant negatively charged particles, with a decay of the electron density towards the PG and a corresponding decay of the negative ion density towards the plasma centre. The understanding of the physics of the complex multi-species $\left(\mathrm{e}^{-}, \mathrm{H}^{-}, \mathrm{H}^{+}, \mathrm{H}_{2}^{+}, \mathrm{H}_{3}^{+}, \mathrm{Cs}^{+}\right)$boundary layer embedded in a 3D magnetic and electric field distribution has improved during the last few years by modelling $[17,26]$ and by dedicated experiments at IPP [8]. A part of this effort is reported in this paper.

\subsection{The BATMAN test facility}

BATMAN was mainly devoted to optimizing the RF source with respect to current density, operating pressure and coextracted electron current. Due to the low pumping speed and capacity of the Ti evaporation pumps and limits in the high voltage supply, the extraction area on BATMAN is limited to $<0.01 \mathrm{~m}^{2}$ and the pulse length to $<6 \mathrm{~s}$. Operation with deuterium is possible for a limited number of pulses.

BATMAN is equipped with the small prototype IPP RF source with one so-called driver; the area of the source body $\left(0.32 \times 0.59 \mathrm{~m}^{2}\right)$ is roughly one-eighth of the ITER source. The depth of the source body is $0.23 \mathrm{~m}$. The driver, where the RF - with a typical power of $50-100 \mathrm{~kW}$ - is coupled to the plasma, is mounted on the back of the source body and consists of a $150 \mathrm{~mm}$ long alumina cylinder with an inner diameter of $235 \mathrm{~mm}$ and a water-cooled RF coil connected to a $1 \mathrm{MHz}$ oscillator. An internal water-cooled Faraday screen protects the alumina cylinder from the plasma. Details can be found in [4].

The plasma expands from the driver into the actual source body, the expansion region, towards the extraction region (see [4]). The latter two are separated by the filter field; created at BATMAN by permanent magnets embedded in a specially designed diagnostic flange. All experiments discussed here are performed with a filter field optimized for sufficient electron suppression in deuterium operation. As the experiments always show that the amount of co-extracted electrons is much larger in deuterium (factor four-to-five for the IPP RF source [8,25]), a larger filter field is needed; the maximum field strength is about $7 \mathrm{mT}$ at the centre of the PG for deuterium, $5 \mathrm{mT}$ for hydrogen. Consequently, the achieved current densities for hydrogen reported in this paper are less than the maximum achievable values at BATMAN [25].

Figure 2 shows a schematic overview of the HV circuit of BATMAN. The ions are accelerated to a calorimeter located at a distance about $1.5 \mathrm{~m}$ from the GG. The source is at a high potential and the regulated $\mathrm{HV}$ voltage is delivered to the grids via a voltage divider with a resistance of the order of $1 \mathrm{kQ}$. The current capability of this voltage divider limits the total voltage at BATMAN to $20-25 \mathrm{kV}^{1}$ An RF transformer separates the source from the RF power supply on ground potential (not shown in the figure).

The electrical currents flowing onto the grids, as well as the current flowing back to the HV power supply, are measured individually. As discussed in detail in [4], currents on the GG, on the calorimeter and the scrapers are solely caused by negative ions. The current accountability is quite good. Typically $10-20 \%$ of the electrically measured ion current $I_{\text {ion }}$ is found on the $\mathrm{GG}\left(I_{\mathrm{GG}}\right)$, depending on the perveance, whereas about $70-80 \%$ of the ion current hits the calorimeter [4]. The current on the EG $I_{\mathrm{EG}}$ can be caused in principle both by

1 The HV system of BATMAN has recently been upgraded by replacing the (unreliable) voltage divider by a second tetrode, allowing pulses of up to $10 \mathrm{~s}$ and a total voltage of $40 \mathrm{kV}$. The necessary upgrade of the extraction system and the calorimeter is part of the mid- to long-term programme of BATMAN. 


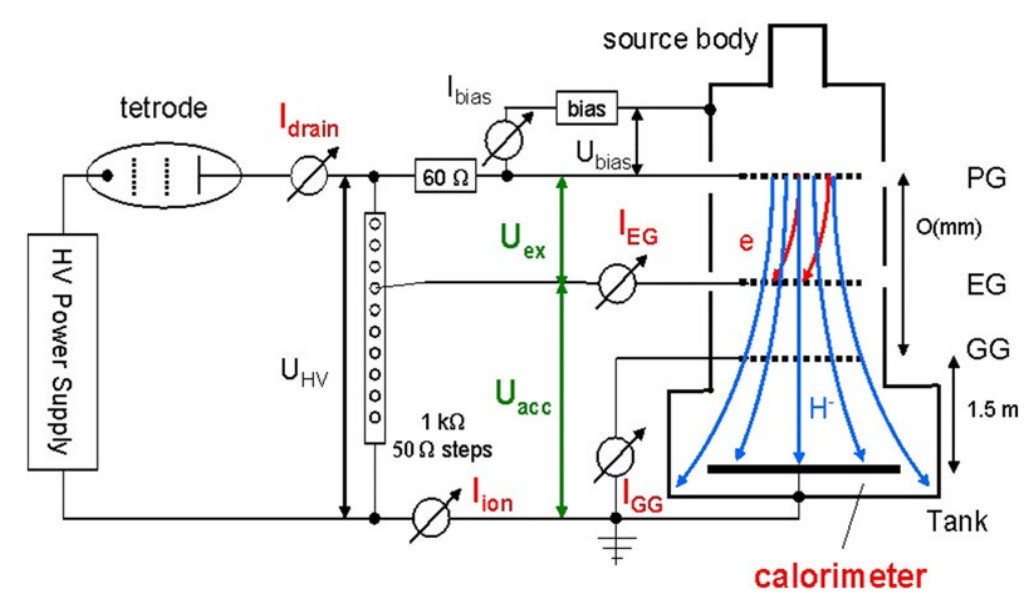

Figure 2. The HV circuit of the BATMAN test bed. The source is at a high potential and the calorimeter is at a distance of $1.5 \mathrm{~m}$ from the GG.

electrons and by negative ions; the ion contribution, however, vanishes for good beam optics. Nevertheless, in the following, the current on the EG is assumed to be solely caused by impinging electrons.

In contrast to [4], in this paper we follow the new ITER current nomenclature: the 'extracted current density' $\left(\boldsymbol{j}_{\text {ex }}\right)$ denotes the amount of negative hydrogen ions that can leave the plasma and is derived from the electrically measured $I_{\text {ion }}$ current; this quantity is a measure of the source performance. The 'accelerated current density' $\left(\boldsymbol{j}_{\text {acc }}\right)$ denotes the amount of negative hydrogen ions that could be (almost) fully accelerated; it is given by the calorimetrically measured current density. The ratio of accelerated to extraction current density is hence a measure of the optical quality of the extraction system.

The calorimeter is a water-cooled copper panel equipped with specially designed thermally isolated areas which are read by thermocouples for beam profile measurements. The measured power density profiles are fitted by a 2D Gaussian and the integration of this Gaussian to infinity gives the total power of negative ions or neutrals not only hitting but also missing the calorimeter. The calorimetric current density is then obtained by dividing this total power by the extraction area and the total beam voltage. With this method, the calorimetric current density gives the lower limit of the accelerated ion current density, as, due to stripping in the extraction system, not all of the ions arrive at the calorimeter with full energy, and some are lost at other beam line components such as the protection scraper of the source flange.

The divergence of the beam is estimated by a comparison of the measured beam profile with a geometrical beam model using the IPP DENSB code [27]. This code assumes beamlets emitted by the GG. Each beamlet has a Gaussian profile where the width of the Gaussian is given by the assumed beamlet divergence, and the amplitude by the amount of power carried by the beamlet. The beamlet divergence is defined as the half $1 / e$-width. Then the power density profile on a certain surface can be calculated by taking the geometry of the GG and the beamline components into account.

As an example, figure 3 shows a comparison of the measured power density profile with the large area grid (LAG) extraction system-having 126 apertures with $8 \mathrm{~mm}$ diameter (see next section) — and the fitted Gaussian with the calculated
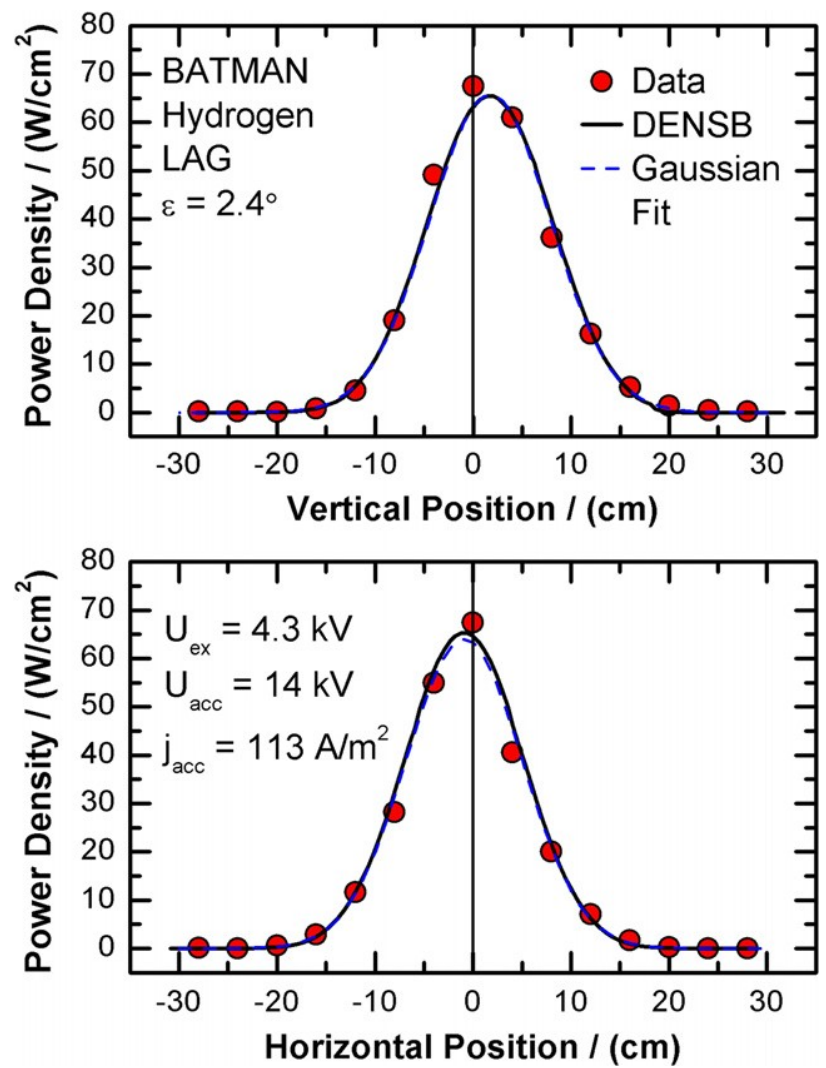

Figure 3. Comparison of measured beam profiles at the BATMAN calorimeter with DENSB calculations for the LAG extraction system, assuming a constant (half $1 / e$ ) divergence $\varepsilon$ of $2.4^{\circ}$ for all 126 beamlets. The Gaussian fit is used to calculate the extracted current density.

DENSB profile. The vertical beam is deflected upwards by a few centimetres due to the interaction of the extracted ions with the magnetic field components of the filter field magnets also present in the extraction system. As the orientation of the magnets in the EG alternates from aperture row to aperture row, no (horizontal) net deflection of the total beam by the electron suppression field occurs.

It is assumed in the DENSB calculations that all beamlets have the same divergence and amount of power, i.e. a 

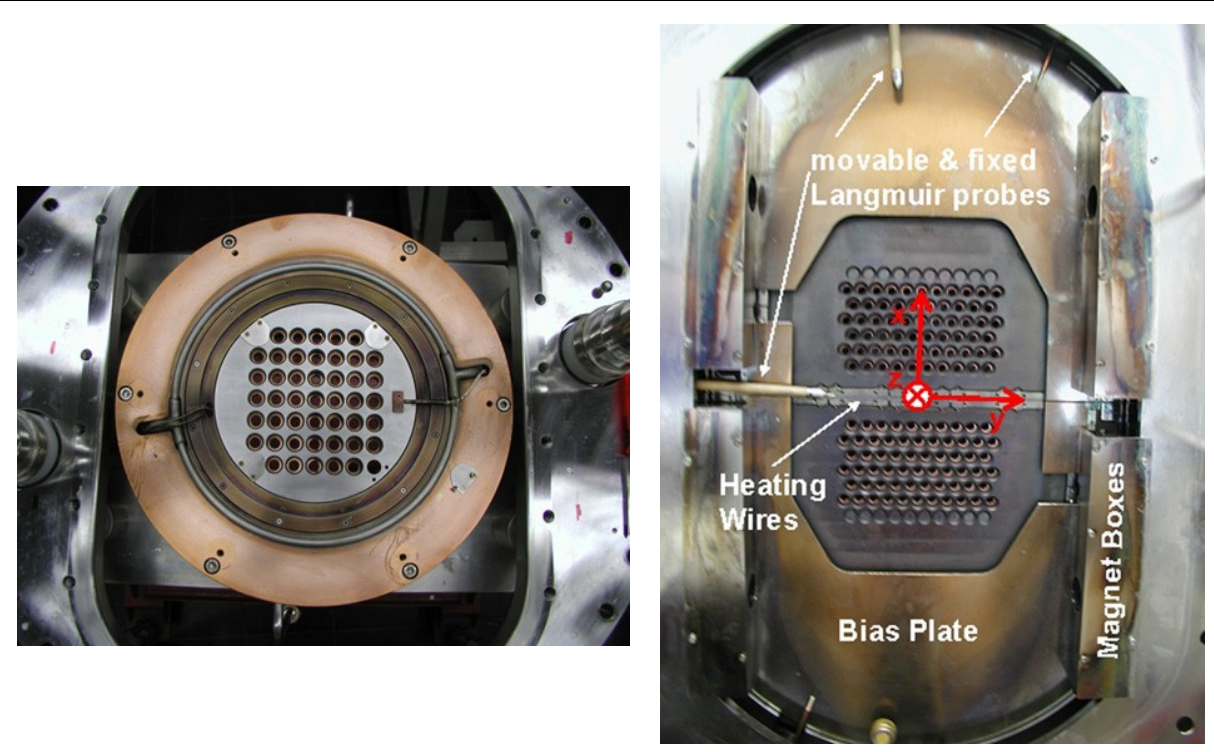

Figure 4. View on the PG of the CEA (left) and the LAG (right) extraction system. The CEA grid has 45 apertures, corresponding to an extraction area of $0.0069 \mathrm{~m}^{2}$. The LAG consists of two identical grid halves with 63 apertures, each corresponding to a total extraction area of $0.0063 \mathrm{~m}^{2}$. BATMAN is equipped with a variety of plasma diagnostic tools, such as movable and fixed Langmuir probes. The coordinate system is indicated for the LAG extraction system, but is also valid for the CEA extraction system.

perfect homogeneous beam. With these assumptions, the measured profile could be reconstructed very well. It might be possible, however, to also reconstruct the measurements by an inhomogeneous divergence and power distribution of the beamlets, but this would impose too many free variables for the fit. Hence, beamlets with equal power and divergence have also been assumed in the following for simplicity, so that the quoted divergences in this paper can be regarded as a mean value for all beamlets.

\subsection{Multi-aperture grid extraction systems at IPP Garching}

Figure 4 shows the photographs of the view onto the PG for the two different extraction systems used in the experiments. The geometry of both systems is shown in figure 5 . Both extraction systems have edge-cooled and electrically heated PGs made of $2 \mathrm{~mm}$ thick molybdenum and actively cooled copper EGs with embedded electron deflection magnets.

The negative hydrogen ion experiments at IPP Garching started in the late 1990s at BATMAN with the so-called CEA extraction system, which was built in cooperation with CEA, Cadarache. The geometry was adopted from the ITER NBI extraction system geometry; the 49 apertures with a diameter of $14 \mathrm{~mm}(\mathrm{PG})$ are arranged in a $7 \times 7$ matrix with a distance of $20 \mathrm{~mm}$ both in the vertical and horizontal direction. The initial extraction gap, i.e. the distance between the plasma and EG, was $3 \mathrm{~mm}$, according to the ITER NBI design value at that time. Due to problems with the power handling of the CEA $\mathrm{EG}$, some of the apertures have been closed, resulting in an extraction area of $0.0067 \mathrm{~m}^{2}$.

Nevertheless, frequent water leaks occurred; hence a new extraction system was built in 2002. This so-called LAG was derived from the IPP positive ion extraction system used for ASDEX Upgrade injection [28] for cost saving reasons. The total extraction area is originally $0.039 \mathrm{~m}^{2}$ and consists of 776 apertures of $8 \mathrm{~mm}$ diameter in the PG. In BATMAN the LAG system must be masked down to an extraction area comparable

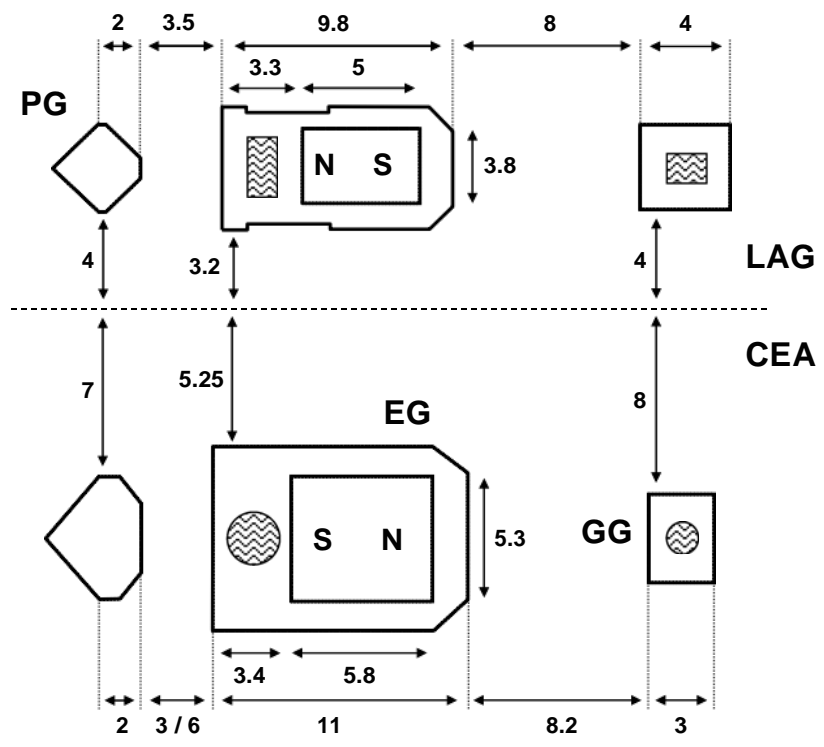

Figure 5. Geometry of the two different extraction systems at BATMAN. The extraction gap of the CEA extraction system was changed from 3 to $6 \mathrm{~mm}$. The cooling channels in the extraction and GG, respectively, are indicated by the waves. Both PGs are edge-cooled, with no embedded cooling channels. Dimensions are given in $\mathrm{mm}$. At the IPP test facilities, the GG is at zero potential; the total voltage is in the range of $20-30 \mathrm{kV}$.

to that of the CEA system because of the limited pumping speed and capacity, but it allowed an easy increase in the extraction area at the long pulse test facility MANITU [29, 30]. The LAG system consists of two identical grid halves with 63 apertures each used for the present experiments; the apertures are arranged in displaced six rows with alternating 11 or 10 apertures with $11.6 \mathrm{~mm}$ distance in the horizontal and $11.9 \mathrm{~mm}$ in the vertical direction. The grid halves are inclined by $0.9^{\circ}$ for beam focusing reasons. The extraction area is, with $0.0063 \mathrm{~cm}^{2}$, very similar to the CEA extraction systems; hence, 


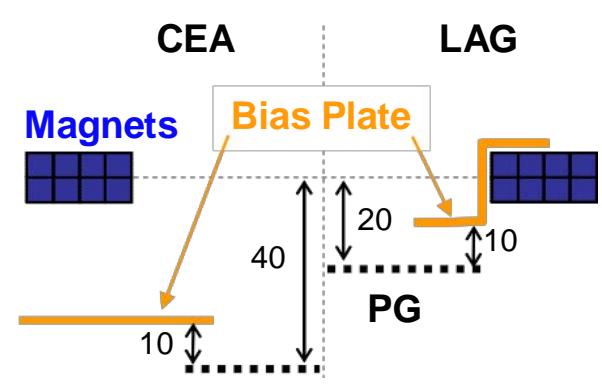

Figure 6. Sketch of the relative axial position of the PG for the CEA grid and LAG system with respect to bias plate and filter field magnets. Dimensions are given in $\mathrm{mm}$. The filter field is created by $2 \times 4$ CoSm magnets with a dimension of $9 \times 13 \times 50 \mathrm{~mm}^{3}$.

the grid conductance, and with that the stripping losses in both systems, are also comparable.

In November 2004, the molybdenum mask on the LAG PG was modified to a plate where the selected holes in the PG are opened by chamfered holes in order to increase the area on the PG for the conversion of neutrals and positive ions to negative ions. With that configuration, the record values reported in [4] have been achieved.

The PG can be biased against the source body. In order to enhance the surface area on source potential near the PG, which is necessary for efficient suppression of the co-extracted electrons [25], this biasing requires, in the case of the LAG, the addition of a so-called bias plate, electrically connected with the source body. The CEA system has this bias plate intrinsically attached, as the smaller extraction system is surrounded by a grid frame that is electrically isolated from the PG, but connected with the source body.

For the experiments reported in this paper, the CEA system was upgraded according to the experience with the LAG system by also connecting a molybdenum mask with chamfered apertures to the PG; furthermore, the power handling of the EG was improved by a rebuild, with a new electrodeposited copper layer on top of the cooling channels. The experiments started with the original $3 \mathrm{~mm}$ extraction gap, but in accordance with the present design of the ITER extraction system, experiments with a $6 \mathrm{~mm}$ extraction gap have also been performed.

Apart from the extraction system geometry, the two extraction systems also differ in the magnetic field structurecaused by different sizes and positions of the magnets embedded in the EG-and in the relative position to the source body-caused by differences in the mounting frame (see figure 6). The distance of the PG in the axial direction to the centre of the filter field magnets for the CEA extraction system is twice the distance as for the LAG system. The relative position of the bias plate to the PG, however, is the same for both extraction systems.

Figure 7 shows the resulting magnetic field strength created by the EG magnets along the aperture centre. At BATMAN, this field is perpendicular to the filter field; both fields are of the same order of magnitude on the plasma side of the PG (about $7 \mathrm{mT}$ at maximum for the filter field). Due to the smaller aperture diameter, the maximum electron deflection field for the LAG extraction system is about twice as large as for the CEA system, both for extraction gaps of 3 and $6 \mathrm{~mm}$. On the other hand, the decay of that field into the plasma is steeper

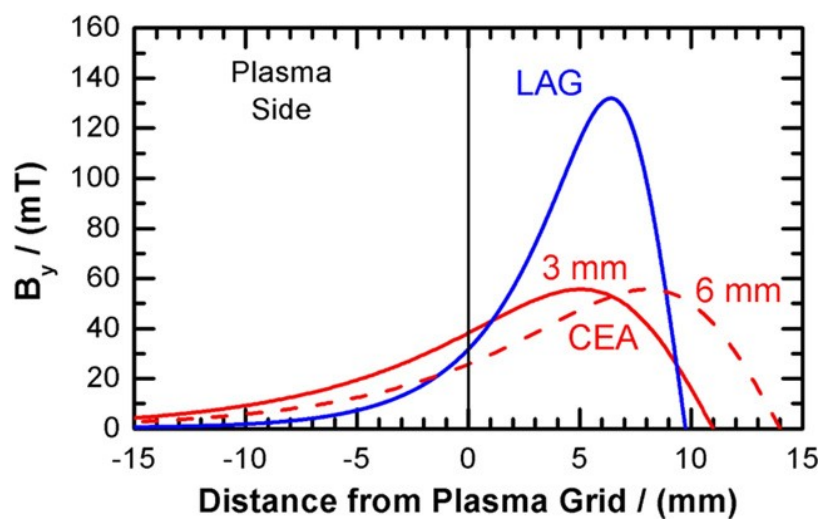

Figure 7. Calculated horizontal magnetic field strength $B_{y}$ of the electron deflection field relative to the PG position for the three different extraction systems (LAG and CEA with 3 and $6 \mathrm{~mm}$ extraction gap, respectively).

in the case of the LAG system. The increase in the extraction gap from 3 to $6 \mathrm{~mm}$ for the CEA system shifts the electron deflection field accordingly, but with minor differences on the plasma side of the PG. In spite of the larger distance between the CEA PG and the filter field magnets, the filter field itself is reduced by only about $10 \%$ at the centre of the PG.

\section{Results and discussion}

The main parameters for the comparison of the source performance at BATMAN are the achieved current densities (extracted and accelerated), the amount of co-extracted electrons, the source efficiency and the beam quality, expressed in the above discussed mean beamlet divergence. The source efficiency is defined as the extracted current density divided by the RF power. By this definition, the almost linear dependence of the extracted ion density on the RF power for a well-caesiated ion source-at least in a certain range and for other optimized parameters such as the bias voltage-is cancelled [4].

As the source performance depends mainly on the caesium conditions such a comparison is not straightforward; after changing the extraction system - by doing this the source has to be vented-it takes some weeks to condition a source in order to achieve optimum performance with caesium. Although one can never be sure that the real optimum was achieved, the 'optimum' performance was defined as achieving a source efficiency of $4 \mathrm{~A} \mathrm{~m}^{-2} \mathrm{~kW}^{-1}$, this being the upper limit both for hydrogen and deuterium for several years for BATMAN [4] ${ }^{2}$.

Due to the fact that the amount of co-extracted electrons in deuterium operation is much larger than in hydrogen (see section 3.2 and $[8,25]$ ), experiments for each extraction system were started with caesium conditioning in hydrogen, in order not to overload the EG. After achieving the optimum performance of $4 \mathrm{~A} \mathrm{~m}^{-2} \mathrm{~kW}^{-1}$, operation was then switched to deuterium. Unfortunately, the deuterium experiments with the CEA extraction system, both for the 3 and the $6 \mathrm{~mm}$ extraction gap, were hampered by technical problems of the HV system

2 In that reference, the maximum achieved source efficiency was quoted to be $3 \mathrm{Am}^{-2} \mathrm{~kW}^{-1}$, but it turned out that during that time the RF power was measured erroneously, being too high by $30-40 \%$. 

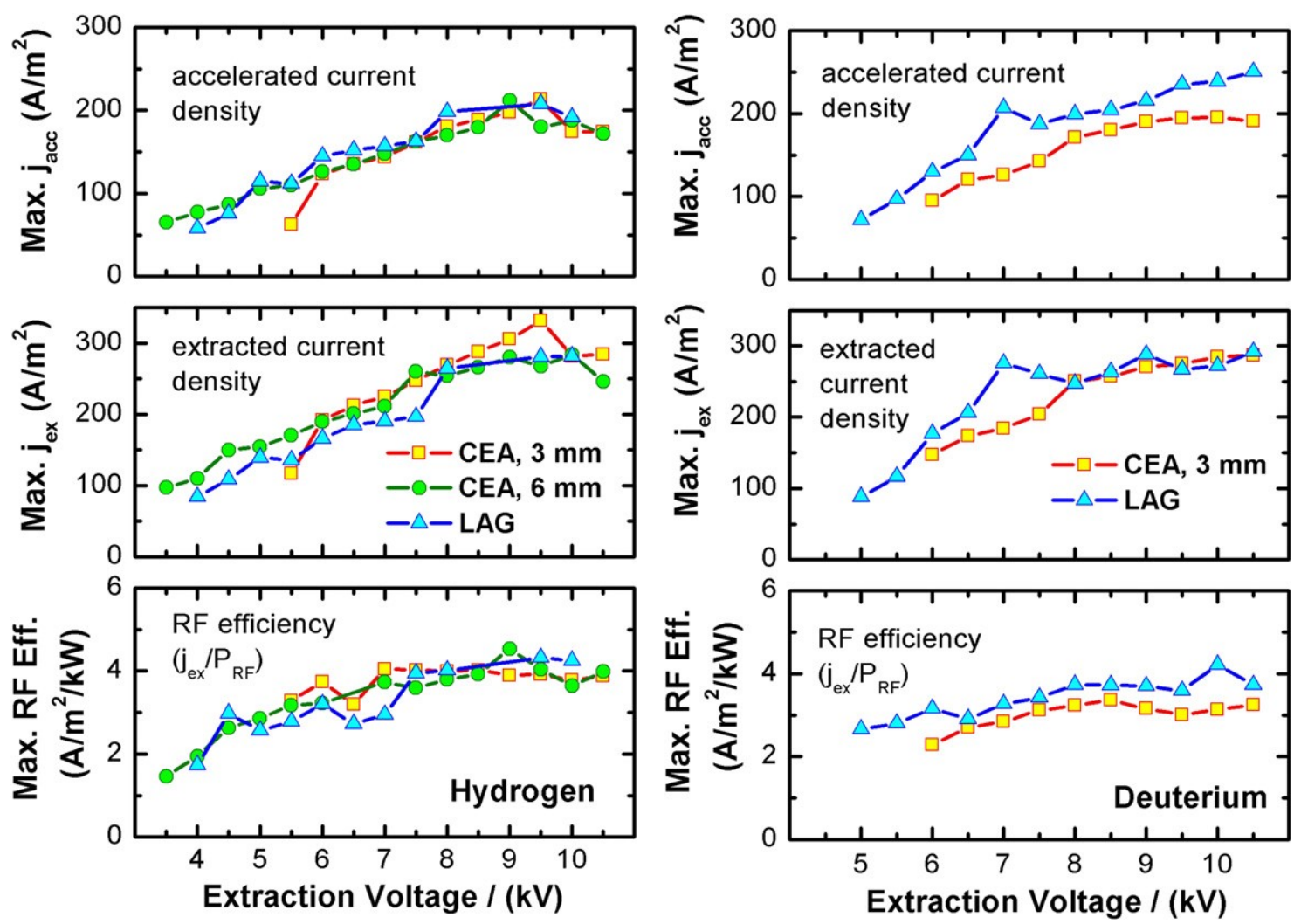

Figure 8. Comparison of the performance of the three different extraction systems. Shown is the overall maximum achieved value of the accelerated current density (top), the extracted current density (centre) and the RF efficiency (bottom), both for hydrogen and deuterium for $0.5 \mathrm{kV}$ intervals of the extraction voltage (the number refers to the upper bound). Deuterium data for the case of the CEA system with a $6 \mathrm{~mm}$ extraction gap are not available for technical reasons.

(frequent failures of the voltage divider, see footnote 1) and of the internal Faraday screen limiting the extraction voltage and the experimental time so that a complete set of data for deuterium could not be achieved.

The main conclusions that are discussed in detail in the next sections are as follows.

- There is no major difference in the source performance regarding the achieved current densities and source efficiency for the different aperture sizes and extraction gaps. This observation is consistent with ion trajectory modelling results.

- There is also no difference in the minimum amount of co-extracted electrons achieved for the different extraction systems, regardless of the differences in the filter field configuration near the PG.

- The beam optics properties of the ITER-relevant CEA extraction system with a $6 \mathrm{~mm}$ extraction gap are better than for the $3 \mathrm{~mm}$ case.

\subsection{Comparison of current density and source efficiency}

Figure 8 shows the comparison of the overall performance of the two extraction systems regarding the current densities and the source efficiency. Data are shown for the experimental periods from October 2005. The initial experiments were with the LAG system. Hence, the data for the LAG system differ slightly from those in [4], so the same measurement (see footnote 2) and source setup is used for comparison. The figure shows the overall maximum extracted and accelerated current density as well as the source efficiency in the database, achieved for certain intervals of the extraction voltage, regardless of other parameters such as RF power and bias voltage for a source pressure below $0.4 \mathrm{~Pa}$. To show the variation of the source performance, the dependence on the extraction voltage was chosen as one could expect that if there are differences between the different extraction geometries, they would be highlighted in this kind of diagram.

It can be clearly seen, however, that within the spread of the experimental data all three cases of extraction geometry (LAG and CEA with 3 and 6 mm extraction gap, respectively) achieve the same current density of accelerated and extracted negative ions in hydrogen operation; the source efficiency is also the same. All extraction systems show the same-almost linear-dependence of the extracted current density on the extraction voltage.

Although the experimental database for deuterium operation is rather limited for the CEA extraction system for the aforementioned technical reasons - that can also be seen in the larger spread of the data, at least for the CEA extraction system with $3 \mathrm{~mm}$ extraction gap, the required extracted current density $\left(290 \mathrm{~A} \mathrm{~m}^{-2}\right.$ ) was achieved.

These results are in clear contrast to the single aperture experiments reported above, where the extracted current density for the $8 \mathrm{~mm}$ aperture was twice as large (280 $\mathrm{A} \mathrm{m}^{-2} \mathrm{D}^{-}$) as for the $14 \mathrm{~mm}$ aperture case [9] under caesium seeded conditions. In these experiments, chamfered 
apertures were also used to increase the extraction probability of negative hydrogen ions. The effects of a possible different gas flow, pressure or stripping losses for the different aperture sizes can be excluded, as no dependence of the extracted current density on the aperture size is seen in pure volume operation.

The difference in the dependence of the extracted current density on the aperture diameter for single and multi-aperture experiments can be explained by the particularities of the processes leading to negative ion generation at the PG surface and the subsequent extraction. The understanding of these processes has been advanced in the last few years by modelling of the boundary layer near the PG at IPP Garching. A PIC code including surface production of negative ions is being developed to study the influence of surface generated negative ions on the properties of the boundary layer [26]. For the typical operational regime of the IPP RF source with large atomic hydrogen density $\left(10^{19} \mathrm{~m}^{-3}\right)$ — compared with the electron and positive ion density $\left(10^{17} \mathrm{~m}^{-3}\right)$ near the grid-a negative space charge builds up in the vicinity of the wall due to the large amount of negative hydrogen ions generated at the grid. As a consequence the probability of the negative ions leaving the PG surface is reduced. Hence, the negative space charge accumulation can be reduced only by increasing the positive ion flux towards the PG.

The negative ions that are created at the PG surface are accelerated into the plasma by the sheath potential and have to be bent back to the aperture for extraction. Their extraction probability can be calculated by the ion trajectory code TRAJAN, also under development at IPP. For details see [18,31]. Briefly, this 3D Monte Carlo code follows each ion that is generated at the PG as it moves through the background plasma and the magnetic and electrical fields near the PG until it is either extracted, hits the PG surface, or is destroyed by collision with electrons and ions. The parameters of the background plasma used are those obtained from measurements. The geometry of the PG with the chamfered apertures and the actual magnetic field patterns (both filter field and electron deflection field) are taken into account.

The first results of the TRAJAN code showed [31] that the extraction probability is in the range of $20-30 \%$ and decreases with increasing ion path length through the plasma near the aperture, i.e. with increasing destruction probability. The path length could be minimized both in experiment and modelling using chamfered apertures - they improve the starting angleand by a low starting energy, i.e. a low sheath potential due to the PG bias voltage. Furthermore, and this is the most important result for this paper, the extraction probability depends primarily on the ratio of the conversion area to the aperture area for otherwise identical plasma parameters and magnetic fields [31]. The conversion area is defined as the area of the PG from which negative ions created by the conversion of impinging hydrogen neutrals and ions can be collected by the aperture(s). It consists of the area of the chamfer surrounding the apertures, as well as the flat areas between the apertures. Although the mean free path of the negative ions in the boundary layer near the PG is of the order of some tens of centimetres [17], the projected range is much shorter due to the movement within the magnetic filter field and the charge exchange collisions. Hence, only negative hydrogen ions created in a certain distance-much smaller than the mean free path-from the aperture can be extracted. But this means that the conversion to aperture area ratio and consequently the extraction probability depends for a single aperture extraction system on the aperture diameter, as seen in the experiments.

In contrast, the ratio of conversion to aperture area is almost independent of the aperture diameter for multi-aperture systems if the grid transparency is the same. This is exactly the case for the extraction systems discussed in this paper-for the LAG extraction system the transparency is $\sim 37 \%$, for the CEA extraction system $\sim 34 \%$; thus the code results explain the observed independence of the extracted current density on the aperture diameter for the IPP extraction systems.

Another important result of the TRAJAN code is that the extraction probability of negative ions does not depend on the electron deflection field components in front of the PG [31]. This result is also supported by the experiments reported in this paper: despite the large differences in the absolute amount and the gradients of the electron deflection field in front of the PG (see figure 7), the extracted current density (and hence the extraction probability) is the same for the different extraction systems.

The TRAJAN code can explain qualitatively the experimental observations, although the calculated amount of the decrease in the current density with increasing aperture diameter (25-33\% reduction for the $14 \mathrm{~mm}$ aperture compared with the $8 \mathrm{~mm}$ aperture $[18,31])$ is still not so large as seen in the experiments (50\% reduction [9]). To understand this is part of the ongoing code development.

As the geometry and hence the transparency of a $5 \times 16$ aperture group of the ITER NBI system [3] is almost the same as the CEA extraction system, it can be expected that the extraction probability of negative ions is the same as at BATMAN for otherwise identical source parameters. To demonstrate that is one of the main motivations of the planned IPP test facility ELISE intended for large-scale extraction from a half-size ITER source.

The same almost linear dependence of the extracted current density on the extraction voltage for the different extraction systems is still not fully understood. One might argue that the linearity might be an artefact of the peculiarities of the experimental run during the caesium conditioning of the source. Normally, during the conditioning phase, the amount of co-extracted electrons decreases and both extraction voltage and RF power would be increased simultaneously, so that both parameters might be linked in the database. Hence, this linear dependence of the extracted current density could be caused by a hidden underlying linear dependence on the RF power, which is always present. This argument might be partially true for large extraction voltages as the source efficiency shows saturation here. But for an extraction voltage below, say, $8 \mathrm{kV}$ this theory of hidden RF power dependence cannot explain the experimental data. Particularly, this linear dependence of the extracted ion current on the extraction voltage is also seen for the same source conditions and for constant RF power; an example is shown in figure 9 for hydrogen operation with the LAG extraction system. Both figures 8 and 9 show that there is an upper limit of the extracted current density for a certain extraction voltage that cannot be exceeded by increasing the RF power, at least for the RF power level available at BATMAN. 


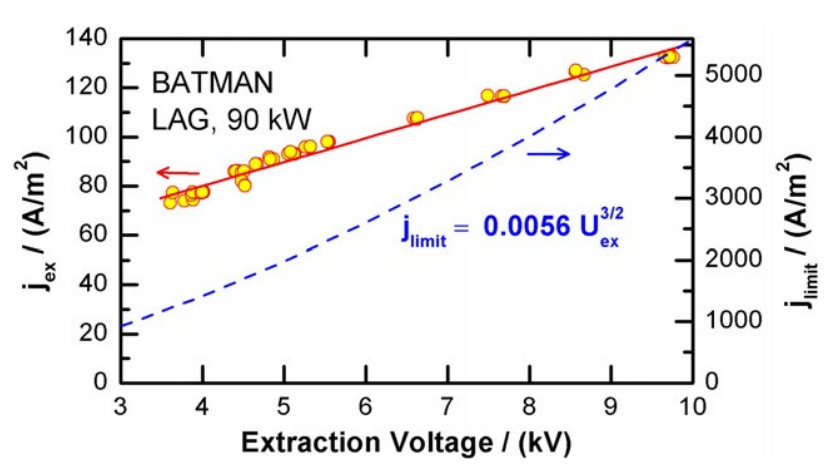

Figure 9. Dependence of the extracted current density on the extraction voltage for a constant RF power for the LAG extraction system. Shown are consecutive pulses with the same source condition in hydrogen operation. This was checked by changing the extraction voltage from 3.5 to $9.8 \mathrm{kV}$ and back to $3.5 \mathrm{kV}$. For comparison, the theoretical curve for the maximal extractable current density, irrespective of the beam quality, for space charge limited extraction according to the Child-Langmuir law using the geometrical PG-EG grid distance, is also shown.

The linear dependence indicates that the extracted current density is not space charge limited as assumed by the ChildLangmuir law $\left(\boldsymbol{j}_{\text {limit }} \sim U_{\mathrm{ex}}^{3 / 2}\right.$, see figure $9, \boldsymbol{j}_{\text {limit }}$ being the maximal extractable current density irrespective of the beam quality). The meniscus shape itself and hence the beam quality is of course determined by the space charge distribution of the ions nearby. Furthermore, the extracted current density is only a few per cent of the space charge limit, indicating that the extraction is limited by the amount of negative ions near the meniscus. As discussed in detail in section 3.3, the optimum beam, i.e. the lowest divergence, is achieved for $4-5 \mathrm{kV}$ for the LAG grid system, the design value. Figure 9 shows that this is achieved at roughly $10 \%$ of the Child-Langmuir limit, a much lower value than achieved in positive ion based systems [32].

A simple explanation for the linear dependence of the extracted current density on the extraction voltage would be that with larger extraction voltage the depth of the meniscus also increases; hence the surface area of the meniscus increases and more negative ions can be extracted, if one assumes a constant negative ion density at the meniscus. But calculations show that this change in the meniscus shape is only minor (see figures 10, 11 and 12) and, most probably, does not explain the observed dependence; this topic is part of the ongoing investigations.

Figure 10 shows an example of ion and electron trajectories for typical extraction parameters $\left(j_{\mathrm{ex}}=\right.$ $188 \mathrm{~A} \mathrm{~m}^{-2}, U_{\mathrm{ex}}=9.6 \mathrm{kV}, U_{\mathrm{acc}}=16.8 \mathrm{kV}$ ) for hydrogen using the KOBRA-3D code [33] for the LAG grid system. This figure shows again that for these parameters the LAG grid system is not optimized.

Figure 11 shows the calculated shape of the meniscus for the three extraction geometries (for slightly different parameters from figure 10). The meniscus, i.e. the $-29 \mathrm{kV}$ line, is located for all systems between 3 and $5 \mathrm{~mm}$. The corresponding potential distributions at the aperture centre are shown in figure 12. For simplicity, the calculations were performed without taking the electron suppression field into account; this however, would change the potentials only

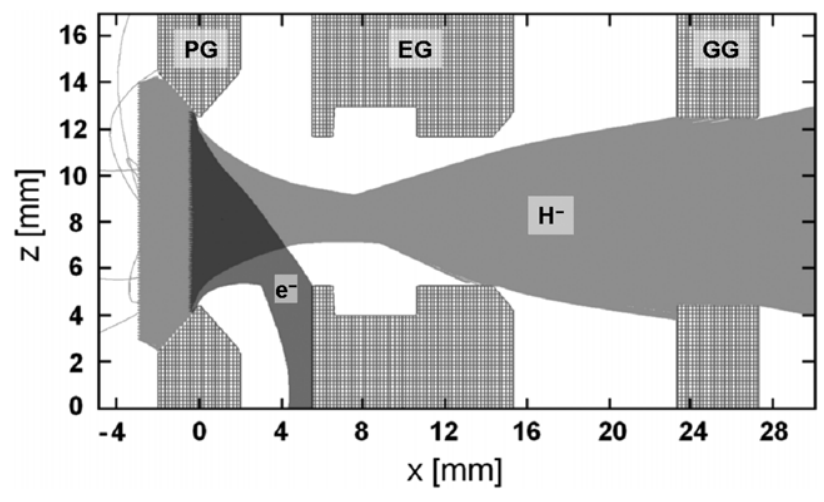

Figure 10. Example of negative ion and electron trajectories for the LAG grid system for an electron and ion current density of $188 \mathrm{~A} \mathrm{~m}^{-2}$ each, an extraction voltage of $9.6 \mathrm{kV}$ and an acceleration voltage of $16.8 \mathrm{kV}$, calculated with the KOBRA-3D code [33].

marginally. Despite the geometric differences, the meniscus shape and potential distribution are similar. In particular, in the case of the CEA extraction system, enlarging the extraction gap from 3 to $6 \mathrm{~mm}$ shifts the potential correspondingly only in the axial direction so that the electric field, i.e. the slope of the potential, in that region is almost constant. This is, at first glance, in contradiction to the fact that the electric field should be halved for the same potential if the distance is doubled. However, the meniscus shape is determined mainly by the aperture geometry for the present extraction systems, where the PG aperture diameter is larger than the extraction gap. This weak sensitivity of the potential distribution on the extraction system geometry also explains the observed similar dependence of the extracted current density on the extraction voltage for the different extraction systems (see figure 8).

\subsection{Comparison of the amount of the co-extracted electrons}

Figure 13 shows the comparison of the three extraction systems regarding the achieved minimum amount of co-extracted electrons. The figure shows the overall minimum achieved ratio of co-extracted electrons to ions for certain intervals of the extracted ion current, regardless of RF power and bias voltage. As for the extracted current density, no major difference in the extraction system performance within the spread of the data could be observed. As mentioned above and reported several times $[8,25]$, figure 13 also shows that the amount of co-extracted electrons is much larger in deuterium than in hydrogen.

The isotope difference in the amount of co-extracted electrons, as well as the understanding of the extraction and suppression of electrons, is in general not very well understood. The electron deflection field has a much larger variation in front of the PG than the filter field for the different extraction systems. The observed independence of the (minimum) amount of co-extracted electrons on the extraction system geometry also indicates that the electron deflection field plays a minor role for the electron suppression, at least for a sufficiently caesiated source. However, as reported in [4], for nonor poorly-caesiated sources, the initial quadrupole magnetic configuration of the LAG extraction system-having almost no field component on the source side of the PG — showed very large currents of co-extracted electrons, leading to subsequent 

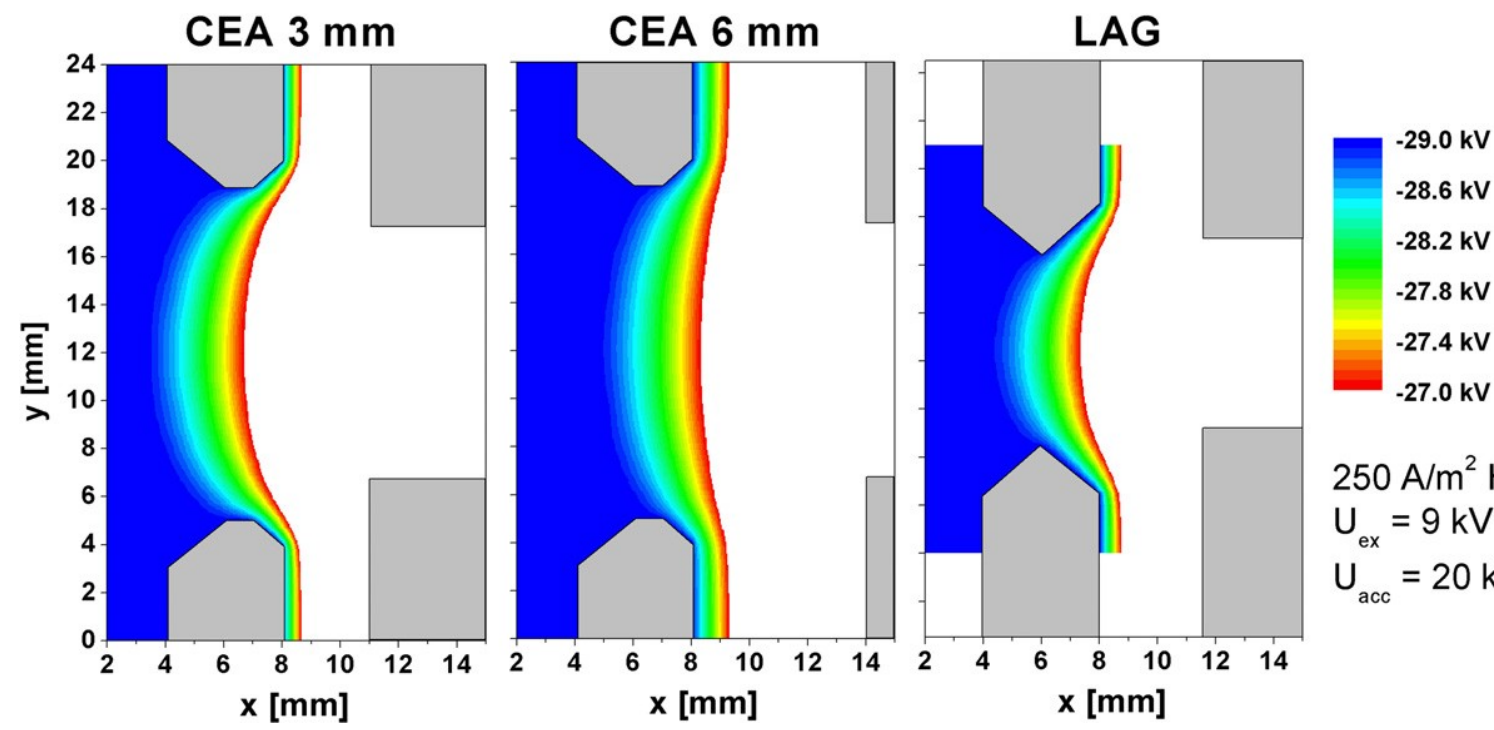

$250 \mathrm{~A} / \mathrm{m}^{2} \mathrm{H}^{-}$

$\mathrm{U}_{\mathrm{ex}}=9 \mathrm{kV}$

$\mathrm{U}_{\mathrm{acc}}=20 \mathrm{kV}$

Figure 11. Calculated equipotential lines for the LAG extraction system and the CEA extraction system with 3 and 6 mm extraction gaps, respectively. Only a small range within $2 \mathrm{kV}$ difference from the total voltage is shown to highlight the meniscus, defined by the $-29 \mathrm{kV}$ line. The coordinate system is chosen so that the PG sits at $4 \mathrm{~mm}$. Plasma is located at the left side.

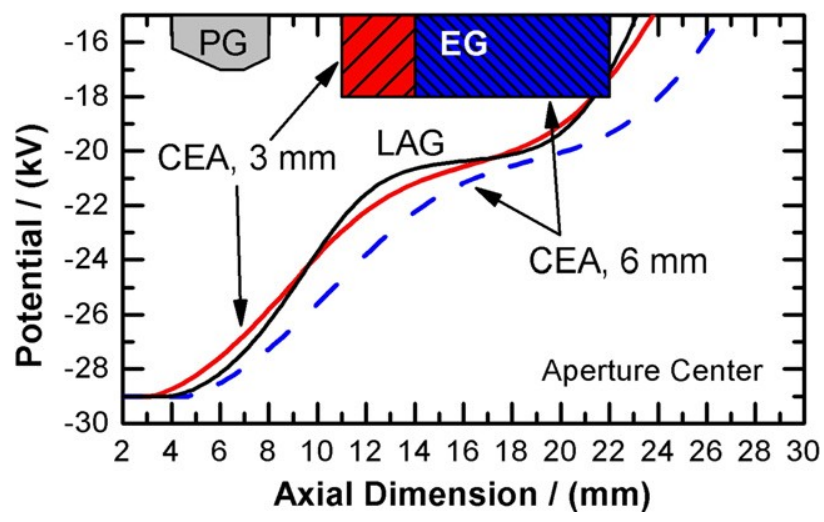

Figure 12. Axial distribution of the extraction potential at the aperture centre for the different extraction geometries (see also figure 10). The positions of the plasma and EG are shown for the CEA extraction system. The electric field, i.e. the slope of the potential, is almost the same for all three-grid systems. The coordinate system is chosen so that the PG sits at $4 \mathrm{~mm}$. Plasma is located at the left side.

damage of the EG. Hence, the electron deflection field may play a different role for the electron suppression in this kind of low pressure negative ion sources, depending on whether the source operates mainly in volume or surface production of negative hydrogen ions, i.e. with a small or large negative hydrogen ion density in front of the PG.

The observed similar amounts of co-extracted electrons for the different extraction systems are also consistent with Langmuir probe measurements performed at BATMAN [8]: the decay of the electron density near the PG is also similar for both the LAG and the CEA extraction system. But this is a consequence of the small differences of the magnetic filter field strength in front of the PG mentioned above, in spite of the fact that the distance of the centre of the filter field magnets to the PG differs by a factor of two. This indicates that the exact position of the filter field is not so important for effective electron suppression. Dedicated experiments at

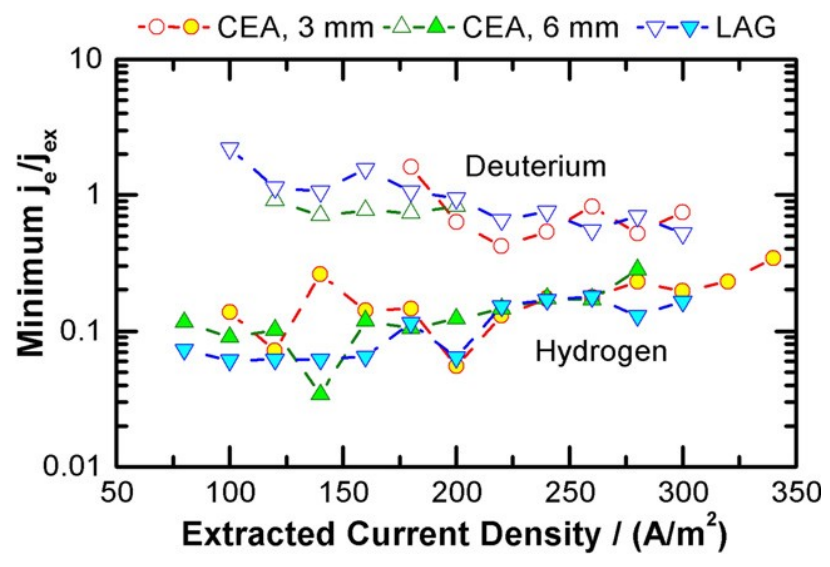

Figure 13. Minimal achieved electron to ion ratio in hydrogen and deuterium for the three extraction systems. The data are the minimum data for intervals of $20 \mathrm{~A} \mathrm{~m}^{-2}$ of the extracted current density. The number refers to the upper bound. The scatter in the hydrogen data is likely by the error of the current measurement for the rather low currents of 0.1-0.2 A only.

BATMAN with more flexibility in positioning the magnetic filter field are part of the next experimental campaign.

\subsection{Comparison of grid optics}

In contrast to the extracted ion and electron current, the beam quality - and hence the ratio of accelerated to extracted current density - does depend on the grid geometry, i.e. on the aperture size and the extraction gap. The latter is indicated in figure 14, where the power density profiles on the BATMAN calorimeter are shown for the CEA extraction system with 3 and $6 \mathrm{~mm}$ extraction gaps, respectively. In both cases the extraction and acceleration voltage, as well as the extraction current density, were the same, but in the case of the $3 \mathrm{~mm}$ extraction gap, the profile is much broader-corresponding to a larger divergence-and much less negative ions hit the calorimeter. 


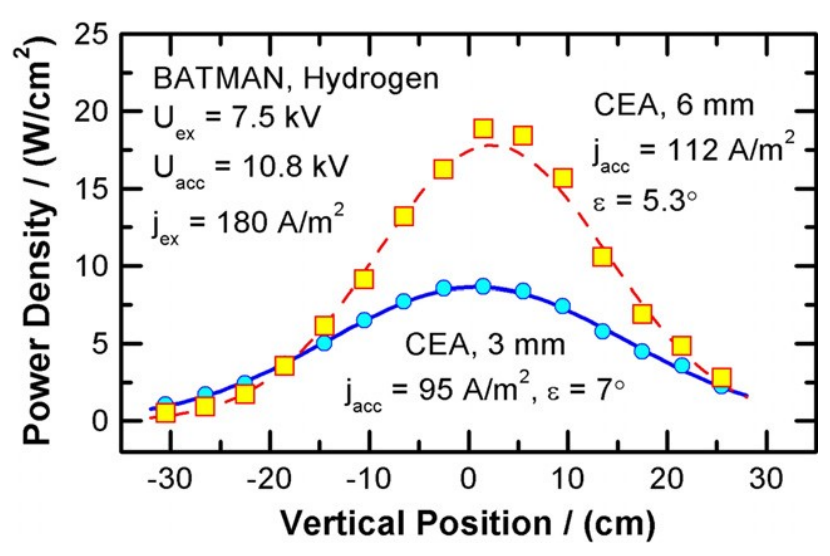

Figure 14. Measured beam profiles at the BATMAN calorimeter for the same voltages and perveance for the CEA grid with 3 and $6 \mathrm{~mm}$ extraction gaps. The divergence $\varepsilon$ is obtained by comparison with the DENSB code calculation, assuming that all beamlets have the same divergence.

The missing ions are most probably lost on the beam scrapers in BATMAN.

Generally, the divergence of a negative ion beam generated with a three-grid extraction system depends on the extracted current, the extraction voltage and the ratio of extraction to the acceleration voltage. It is commonly assumed that the negative ions are ejected normally to the meniscus surfaceas determined by the Child-Langmuir law-and form a convergent beamlet until they diverge due to the space charge expansion. The latter happens especially in the long, potential free extraction aperture. The beamlet then converges again due to the acceleration voltage. The final beam divergence then depends rather critically on the grid geometry for given potentials and currents as ion optics calculations show.

The beam codes used for negative ion extraction are up to now adopted from positive hydrogen ion codes by reversing the polarity. But as discussed above, the shape of the meniscus may be different for positive and negative hydrogen ion extraction for otherwise identical potentials due to the different mass of the space charge compensating particles (electrons or positive ions). These processes are already included in the more sophisticated codes such as KOBRA-3D [33]. Furthermore, the space charge blow-up within the extraction system and in the drift region towards the calorimeter is critical. This is especially true for low-voltage acceleration experiments such as BATMAN, where the ions have a low velocity. The space charge expansion and hence the beam width on the calorimeter depend on the tank pressure due to the fact that the space charge compensation is done by positive ions created downstream by the interaction of the beam with the residual gas in the extraction and drift regions.

Comparisons of the beam optic model results with the measured beam profiles need to be interpreted carefully, at least for low-voltage experiments. Attempts to include these beamline effects in the models are underway. On the other hand, experiments and beam code calculations agree very well for beams with energies near $1 \mathrm{MeV}$ [34]; at these high energies, space charge effects can be neglected [35].

Figure 15 shows the minimum mean beamlet divergence achieved for the LAG extraction system and the CEA extraction system with a $6 \mathrm{~mm}$ extraction gap, which is also currently foreseen for the ITER NBI extraction system [3]. The figure shows the dependence on the extraction voltage and also on the ratio of extraction to acceleration voltage. Again, the overall minimum beamlet divergence in the database is shown, achieved for certain intervals of the extraction voltage and the acceleration to extraction voltage ratio.

The LAG extraction system shows an optimum divergence of about $2^{\circ}$ for an extraction voltage of $4-5 \mathrm{kV}$ and a voltage ratio in the range of 3.5-4.5. Similar minimum divergences have been measured at the MANITU test facility, which is also equipped with the LAG extraction system for similar voltages by Doppler shift $\mathrm{H}_{\alpha}$ spectroscopy [8]. This voltage range for achieving the minimum divergence corresponds to the design values of the LAG extraction system: the geometry was optimized for a $200 \mathrm{~A} \mathrm{~m}^{-2}$ deuterium beam with $5.4 \mathrm{kV}$ extraction and $27 \mathrm{kV}$ acceleration voltage [28]. The low extraction voltage was also intended for use in the ITER NBI system at that time (late 1990s). As the divergence scales roughly with the square root of the voltage, the $2^{\circ}$ divergence at BATMAN corresponds to about $2^{\circ} \times(1 \mathrm{MeV} / 20 \mathrm{keV})^{0.5} \approx$ 5 mrad divergence for a $1 \mathrm{MeV}$ beam; that is within the required range of divergences for the ITER NBI system [9].

However, all the recent experiments have shown (see [4-8] for the RF source, [16] for the filamented source, and also figure 8) that an extraction voltage of $9-10 \mathrm{kV}$ is needed to achieve the required extracted ion current density. Hence, the experiments with the LAG extraction system were performed mostly in this range of extraction voltage. But due to the technical limit of the total voltage at BATMAN (20-25 kV), the ratio of acceleration to extraction voltage must be kept below 1.5, resulting in poor quality beams (see figure 10). The same limit for the available acceleration voltage also holds for the CEA extraction system at BATMAN, but this extraction system with the $6 \mathrm{~mm}$ extraction gap is optimized for $9 \mathrm{kV}$ extraction voltage. The minimum achieved divergence for the $6 \mathrm{~mm}$ extraction gap is indeed better by about $1^{\circ}$ than for the LAG extraction system at $9 \mathrm{kV}$, but still rather high due to the limits in the acceleration voltage.

The experiments show that the minimum divergence, as well as the differences in the optics of the different extraction systems predicted by code calculations, agree qualitatively with the measurements - the voltage range of the optimum achieved divergence agrees with the design expectationsso that there is some confidence that for an ITER-relevant extraction system geometry, i.e. identical to the CEA $6 \mathrm{~mm}$ extraction gap, a beam can be produced with a sufficiently low divergence, by having enough acceleration voltage. To demonstrate this is also a main task of the new IPP test facility ELISE, which will be equipped with a three-grid acceleration system with ITER-relevant geometry (14 mm diameter, chamfered apertures and $6 \mathrm{~mm}$ extraction gap) and with an acceleration voltage of up to $50 \mathrm{kV}$.

\section{Conclusions}

Experiments at the IPP test facility BATMAN with different small multi-aperture extraction systems-having an extraction area of $0.006-0.007 \mathrm{~m}^{2}$ - showed that in contrast to single aperture experiments, the extracted current density does not depend on the aperture diameter if the grid transparency is the 


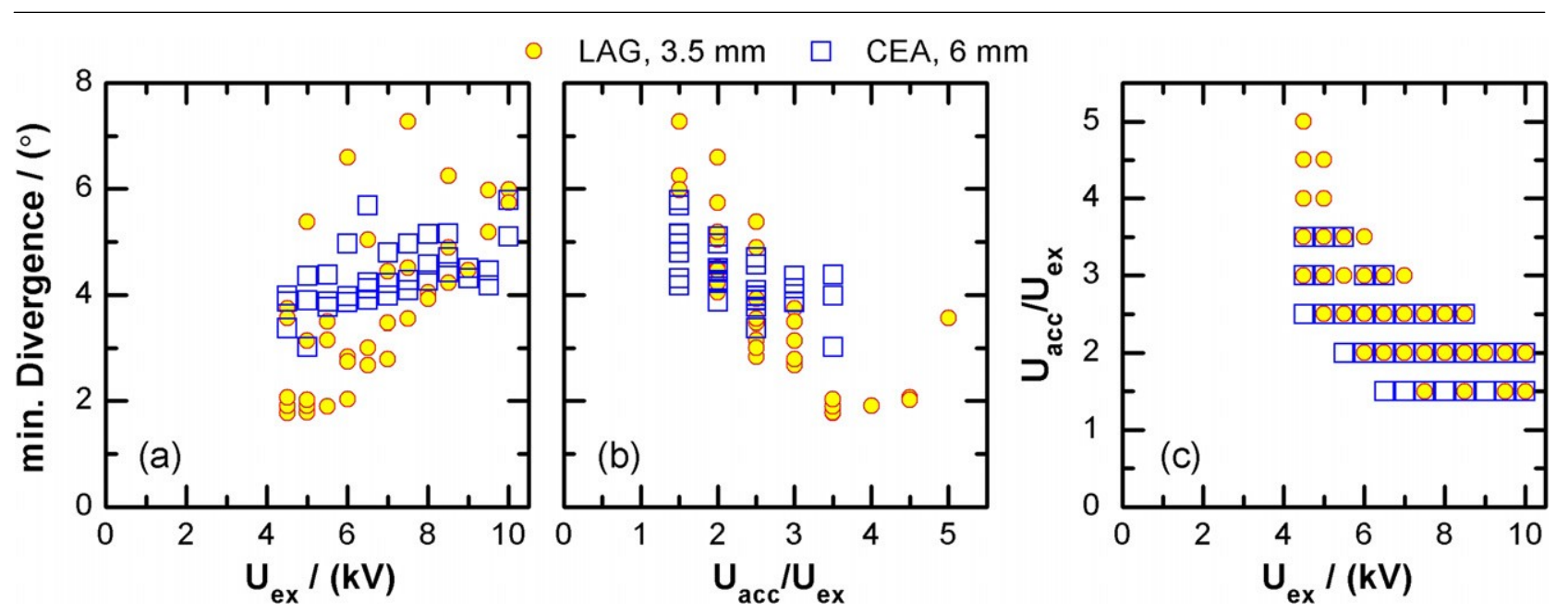

Figure 15. Dependence of the minimum achieved divergence for the LAG extraction system and the CEA, $6 \mathrm{~mm}$ extraction system on the extraction voltage $(a)$ and on the ratio of extraction to acceleration voltage $(b)$ for certain intervals of these parameters. The numbers refer to the upper bounds of the interval. Graph $(c)$ shows the range of the possible voltage ratio for a given extraction voltage, as the total voltage at BATMAN is limited to $20-25 \mathrm{kV}$.

same. This corresponds to results of ion trajectory calculations of the extraction probability for negative hydrogen ions being generated at the plasma grid. As the ITER extraction system transparency is rather similar to the used small IPP extraction systems, it can be reasonably expected that the extraction probability is similar to that of the small experiments, if plasma parameters and the magnetic field configurations near the plasma grid are similar.

In contrast to the extracted current density, the beam quality-and hence the accelerated current density-does depend on the grid geometry, i.e. on the distance between the plasma and extraction grid. Due to limits in the available voltage range at BATMAN, the optimum divergence for the ITER-relevant extraction system could not be achieved, but an increase in the distance of the extraction grid from the plasma grid-as was also done in the last few years in the design of the ITER extraction system-decreased the divergence considerably.

In conclusion, the experiments reported in this paper support the present design of the geometry of the extraction system of the neutral beam system of ITER.

\section{Acknowledgments}

The work was supported by a grant from the European Union within the framework of the EFDA (European Fusion Development Agreement). The authors are solely responsible for the content.

\section{References}

[1] Hemsworth R. et al 2008 Rev. Sci. Instrum. 79 02C109

[2] Schunke B. et al 2009 Proc. 1st Conf. on Negative Ions, Beams and Sources (Aix-en-Provence 2008) AIP Conf. Proc. 1097 $480 \mathrm{http} / / /$ www.virtualjournals.org/getpdf/servlet/ GetPDFServlet?filetype=pdf\&id= APCPCS001097000001000480000001\&idtype $={ }_{\text {cvips }}$

[3] Hemsworth R. et al 2009 Nucl. Fusion 49045006

[4] Speth E. et al 2006 Nucl. Fusion 46 S220

[5] Franzen P. et al 2007 Nucl. Fusion 47264
[6] Fantz U. et al 2007 Plasma Phys. Control. Fusion 49 B563-80

[7] Stäbler A. et al 2009 Fusion Eng. Des. 84 265-8

[8] Fantz U. et al 2009 Nucl. Fusion 49125007

[9] de Esch B. et al 2009 Proc. 1st Conf. on Negative Ions, Beams and Sources (Aix-en-Provence 2008) AIP Conf. Proc. 1097 309 http://www.virtualjournals.org/getpdf/servlet/ GetPDFServlet?filetype=pdf\&id= APCPCS001097000001000309000001\&idtype $={ }_{\text {cvips }}$

[10] Krylov A. et al 2006 Fusion Eng. Des. 81 2239-48

[11] de Esch H.P.L., Hemsworth R.S. and Massmann P. 2005 Fusion Eng. Des. 73 329-41

[12] Inoue T. et al 1995 Rev. Sci. Instrum. 663859

[13] Tsumori K. et al 1995 Fusion Eng. Des. 26473

[14] Takeiri Y. et al 2010 Rev. Sci. Instrum 81 02B114

[15] Massmann P. et al 2000 Nucl. Fusion 40589

[16] Trainham R. et al 1998 Rev. Sci. Instrum. 69926

[17] Takado N. et al 2006 Rev. Sci. Instrum. 7703 A533

[18] Gutser R. et al 2009 Proc. 1st Conf. on Negative Ions, Beams and Sources (Aix-en-Provence 2008) AIP Conf. Proc. 1097 297 http://www.virtualjournals.org/getpdf/servlet/ GetPDFServlet?filetype=pdf\&id= APCPCS001097000001000297000001\&idtype $=$ cvips

[19] Fantz U. et al 2006 Nucl. Fusion 46 S297-S306

[20] Masiello A. et al 2009 Fusion Eng. Des. 84 1276-80

[21] Franzen P. et al 2009 Proc. 1st Conf. on Negative Ions, Beams and Sources (Aix-en-Provence) AIP Conf. Proc. 1097451 http://www.virtualjournals.org/getpdf/servlet/ GetPDFServlet?filetype $=$ pdf\&id $=$ APCPCS001097000001000451000001\&idtype $=$ cvips

[22] Heinemann B. et al 2009 Fusion Eng. Des. 84 915-22

[23] Marcuzzi D. et al 2009 Proc. 25th Symp. on Fusion Technology (Rostock, Germany, 2008) Fusion Eng. Des. 84 1253 doi:10.1016/j.fusengdes.2008.12.084

[24] Christ-Koch S. et al 2009 Plasma Sources Sci. Technol. 18025003

[25] Franzen P. et al 2008 AIP Conf. Proc. 99351

[26] Wünderlich D. et al 2009 Plasma Sources Sci. Technol. 18045031

[27] Penningsfeld F.P. 1986 Technical Report IPP 4-229

[28] Heinemann B. et al 1999 Proc. 20th Symp. on Fusion Technology (Marseille, France, 1998) Fusion Eng. Des. 46 433

[29] Kraus W. et al 2008 Rev. Sci. Instrum. 79 02C108

[30] Kraus W. et al 2009 Proc. 1st Conf. on Negative Ions, Beams and Sources (Aix-en-Provence 2008) AIP Conf. Proc. 1097 
275 http://www.virtualjournals.org/getpdf/serv1et/ GetPDFServ1et?filetype=pdf\&id=

APCPCS001097000001000275000001\&idtype $=$ cvips

[31] Gutser R. et al2009 Plasma Phys. ControL Fusion 51045005
[32] Surrey E. and Ho1ms A.J.T. 1990 Nucl. Instrnm. Methods Phys. Res. A 298 383-8

[33] Spadtke P. and Wipf S. 1989 GSI Report 89-09

[34] Svensson L.et al 2006 Nucl. Fusion 46 S369-78

[35] Franzen P. et al 2003 Fusion Sci. Techno[ 44 776-90 